\title{
Erich L. Lehmann's Work on the History of Classical Statistics
}

\author{
Javier Rojo
}

The papers in this chapter present a fascinating collection of insights and historical facts about the gestation and early periods of classical statistics. Erich, having played a very important role in these developments, is able to masterfully interweave his memoirs with information obtained trough careful readings of the correspondence between some of the other major players in these developments. Although all the papers are brought together by common themes, they can roughly be classified into two groups. One group of papers go to the root of some of the important breakthrough ideas and concepts in classical statistics; their origin, evolution, and impact. In the second group of papers, Erich in a somewhat more leisurely style, describes the personalities and the history of events at the end of the $19^{\text {th }}$, and beginning of the $20^{\text {th }}$, century that led to the development of statistics.

The earliest paper in the first group, Lehmann (1958), is being published here for the first time. It discusses the interpretation of confidence statements beginning with Lapace (1812). It is here that Erich found the first expression that may be interpreted as a confidence statement:

$$
P\left\{\frac{-T \sqrt{2 i(n-1)}}{n \sqrt{n}} \leq p \leq \frac{T \sqrt{2 i(n-1)}}{n \sqrt{n}}\right\}=\frac{2}{\sqrt{\pi}} \int_{0}^{T} e^{-t^{2}} d t,
$$

where $i$, in Laplace's notation, indicates the number of successes in $n$ independent and identical Bernoulli trials with probability of success $p$. Erich includes the original paragraphs in French and states that, although Laplace did not provide an interpretation for (1), the use of this expression by him and others indicated that the interpretation was that of $p$ falling in the interval defined by the fixed left and right limits, and with the probability given, in (1). Furthermore, Lehmann (1958) states, Poisson incorporated (1) into his own work, Poisson (1837), and developed a fiducial distribution for $p$.

\footnotetext{
J. Rojo

Professor of Statistics, Department of Statistics, Rice University, Houston, TX 77005

e-mail: jrojo@rice.edu
} 
Lehmann (1958) presents Poisson's original discussion in French, with some changes in notation and some minor annotations. Several other authors discussed (1), but it is not until 1927 that Erich discovered the first proper (frequentist) interpretation of (1) by E. B. Wilson.

Extensions to the estimation of the difference of two binomial probabilities are also discussed and Erich concludes the paper with the following paragraph:

\begin{abstract}
It is the achievement of Laplace and Gauss, and of their followers throughout the $19^{\text {th }}$ century, to have recognized the importance of obtaining probability limits for unknown parameters. They also developed a method for determining such limits, which is free of a priori assumptions regarding these parameters: The inversion of direct probabilities statements based on large sample approximations. However, it appears to have been understood only in the present century that in the resulting statements the parameter is constant and the probability refers to the variation of the limits.
\end{abstract}

Lehmann (1990) addresses the question: Where do models come from? Examining what Fisher and Neyman had to say in this regard, Erich concludes that Fisher did not envision a general theory of model selection, preferring to leave this task to others ("the practical statisticians"), and adds that Fisher later offered some general advice: (i) use those models that are familiar ("and know how to handle") while (ii) understanding that the parsimony of the selected model will depend on the "volume of data". By contrast, Neyman had a constant interest in the theory of modeling. Among the important Neyman's contributions to this area, Erich writes, was his recognition of the existence of two classes of models: "explanatory" and "interpolating". The former type needs to be informed by the available knowledge about the scientific question at hand, while the latter type is more concerned with selecting models that provide a good fit to the data. Addressing objections from the applied statistician camp that stated "that they know exactly where their models come from", Erich asks the provocative question:

\begin{abstract}
Is applied statistics, and more particularly model building, an art with each new case having to be treated from scratch (although even artistic endeavors require techniques which can be systematized and learned), completely on its own merits, or does theory have a contribution to make to this process?
\end{abstract}

Erich then proceeds to discuss three general ways in which theory can contribute in this regard: (i) by providing a "reservoir of models"; (ii) by developing techniques for choosing a model from a restricted class; (iii) by classifying models, e.g. explanatory vs. empirical, that can lead to a better understanding of its applicability. The paper ends with some comments on areas that, it is stated, required further work. For example, the assumption of independence. It is also suggested that ideas external to statistics, e. g. from artificial intelligence, may contribute to model selection.

In Lehmann (1993a), Erich examines the question of who should be credited for inspiring the development of the Neyman-Pearson theory. Through his incisive writing, Erich takes the reader to the end of the $19^{\text {th }}$, and beginning of the $20^{\text {th }}$, century to a debate between Bertrand and Borel on a probabilistic approach to testing. At play, are the conflicting versions of Neyman and Pearson as to the source of their inspiration. It is pointed out by Erich that, while Neyman credited Borel's debate with Bertrand, and in particular the thoughts expressed by Borel, for his inspiration, Pearson credited 
Gosset. In trying to resolve the issue, Erich points out that a letter from Gosset to Pearson included a crucial suggestion:

If there is an alternative hypothesis which will explain the occurrence of the sample with more reasonable probability ... you will be very much more inclined to consider that the original hypothesis is not true.

After a careful review of the correspondence from Neyman to Pearson, Erich concludes:

... there appears to be no mention of Borel in this or other letters of Neyman's to Pearson during this period. This combination of circumstances lends some support to Pearson's recollections.

The motivation behind Lehmann (1999) is the following statement by F. N. David, as discussed in Laird (1989), that Erich found very intriguing:

I think he [Gosset] was really the big influence in statistics ... . He asked the questions and

Pearson and Fisher put them into statistical language and then Neyman came to work with the mathematics. But I think most of it stems from Gosset.

Erich then goes on to state "The aim of this paper is to consider to what extent David's conclusion is justified." The paper examines three different aspects of Gosset's contributions to small-sample methodology: (i) Distributional problems, (ii) Robustness against departures from normality, (iii) The choice of a test. Through the journey of Erich's analysis of the correspondence of Fisher, Gosset, and Pearson, a completely new image of Gosset comes alive. Although Gosset, by his own admission was unable to solve some important problems due to his lack of mathematical preparation, he is now seen in his new role of pointing both Fisher and Pearson in the direction of important statistical issues that required attention. Gosset is not afraid to push Fisher on these issues and does not back down even when Fisher pushes back.

Erich concludes by agreeing with F. N. David's assessment regarding Pearson. Regarding Fisher, although Gosset exerted influence on some of Fisher's work, Fisher's contributions were numerous and many did not benefit from Gosset's insights. Thus F. N. David's assessment does not apply to Fisher. Finally, Neyman, through Pearson - see Lehmann (1993a), was influenced by Gosset in the development of the Neyman-Pearson theory, although as discussed earlier, Neyman credited Borel for his inspiration.

In Lehmann (2008a), Erich undertakes a thorough critique of the underlying assumptions of the linear model. The three aspects of the model that are the subjects of this scrutiny are the assumptions of: (i) normality; (ii) independence; and (iii) the linear structure in the model. Whereas assumption (i) has received more than its share of attention, (ii) and (iii) have not. Furthermore, Erich writes, the types of data that arise in the various areas of research, (e.g. physical, biological, and social sciences), are quite different and present different challenges. As the paper was published in a volume in honor of David A. Freedman, Erich concludes with a discussion of Freedman's strong criticism of the misuse of the linear regression methodology.

In the last paper in the first group, Lehmann (2009), Erich examines the history of optimality. From Laplace's and Gauss' work on best linear estimates of parameters, 
through Fisher's work on maximum likelihood estimation and the Neyman-Pearson developments, Wald's decision theory, and the Hunt-Stein theorem and its applications, Erich's clear and concise narrative summarizes most of the important developments in optimality. After discussing some extensions of the Neyman-Pearson theory to for example - sequential analysis, robustness, and multiple testing, and after briefly discussing Kiefer's work on optimal experimental design, Erich briefly touches on some criticisms of optimality (e.g. Tukey's). The paper concludes by stating that, as it regards to the state of optimality today,

Ad hoc solutions are proposed and tried out on a few examples. This is a natural first step, but eventually we will want to justify the solutions at which we have arrived intuitively and by trial and error. A theoretical underpinning will be provided and the conditions will be found under which these solutions are the best possible.

The second group of papers includes a brief account of young Erich's immediate family and the tragic circumstances that led the family to leave Germany and settle in Switzerland. After this passage, in a conversation masterfully orchestrated by DeGrot (1986), Erich provides a more leisurely expedition into his career that started in Switzerland after his father suggested mathematics as a field of study instead of German literature which is what Erich felt a passion for. After five years in Switzerland, Erich attended Trinity College in Cambridge for two years and then went to New York where he was ushered by Courant to Berkeley where he spent close to sixty eight years. Together with Lehmann (1993b), Lehmann (1996), and Lehmann (2008b), these fascinating accounts of Erich's professional career immerse the reader in the developments of statistics in The United States during the $20^{t h}$, and early part of the $21^{s t}$, century. The portraits of the major personalities in these developments and in Erich's career come to life through Erich's pen. The origin and development of the Statistics Department in Berkeley is wonderfully chronicled as is the broader picture of the development of statistics. In addition, Lehmann (1997) succeeds in conveying well the excitement, success and tribulations of being a successful textbook writer. Finally, Lehmann (2004) which appeared in a volume of papers presented at the inaugural Lehmann Symposium, discusses the connection between optimality and symposia embodied in the person of Jerzy Neyman, who was the originator of the Berkeley Symposia and played a major role in the development of optimality theory. This paper contains a facsimile of a letter from Neyman to Pearson that indicates that Neyman had not, at that point, yet reached the final solution to the problem. An excerpt of a letter dated two weeks later, however, confirms that the solution is finally, for all practical purposes, at hand. The origin, life, strong legacy, and death of the Berkeley Symposium is also discussed. The paper ends with Erich's blessing of the "new" Lehmann Symposium:

We may consider Neyman to be the godparent of this symposium, both through his founding of optimality theory and the inspiration provided by his symposia.

The fourth Lehmann Symposium recently took place at Rice University during May of 2011. Plans are currently under way for a Lehmann Centennial in 2017. 


\section{References}

[1] M. H. DeGroot. A Conversation with Erich L. Lehmann. Statist. Sci., Vol. I, 243-258, 1986.

[2] P. S. Laplace. Théorie analytique des probabilités. Paris. 1812.

[3] E. L. Lehmann. Some Early Instances of Confidence Statements. Statistical Laboratory, University of California, Berkeley. ONR 5 Technical Report to the Office of Naval Research. Unpublished. September 1958.

[4] E. L. Lehmann. Model specification: The views of Fisher and Neyman, and later developments. Statist. Sci., Vol. 5, pp. 160-168, 1990.

[5] E. L. Lehmann. The Bertrand-Borel Debate and the origins of the Neyman-Pearson theory. In Statistics and Probability. A Raghu Raj Bahadur Festschrift, (Ghosh et al Eds.), pp. 371-380. Wiley Eastern Ltd. New Delhi, 1993a.

[6] E. L. Lehmann. Mentors and early collaborators: reminiscences from the years 1940-1956 with an epilogue. Statist. Sci., Vol. 8, pp. 331-341, 1993 b.

[7] E. L. Lehmann. The creation and early history of the Berkeley statistics department. In Festschrift for David Blackwell, IMS Lecture Notes - Monograph Series, Vol. 30, (T. Ferguson, Ed.), pp. 139-146, 1996.

[8] E. L. Lehmann. Testing statistical hypotheses: The story of a book. Statist. Sci. Vol. 12, pp. 48-52, 1997.

[9] E. L. Lehmann. 'Student' and small-sample theory. Statist. Sci., Vol. 14, pp. 418-426, 1999.

[10] E. L. Lehmann. Optimality and symposia: Some history. In The First Erich L. Lehmann Symposium - Optimality. (V. Perez-Abreu and J. Rojo, Eds.), IMS Lecture Notes-Monograph Series, Vol. 44, pp. 1-10, 2004.

[11] E. L. Lehmann. On the history and use of some standard statistical models. In Probability and Statistics: Essays in Honor of David A. Freedman, (D. Nolan and T. Speed, Eds), IMS Collections Vol. 2, pp. 114-126, 2008a.

[12] E. L. Lehmann. Reminiscences of a Statistician: The Company I Kept, Springer, 2008b.

[13] E. L. Lehmann. Some history of optimality. In The Third Erich L. Lehmann Symposium Optimality, (J. Rojo, Ed.) IMS Lecture Notes-Monograph Series, Vol. 57, pp. 10-17, 2009.

[14] S. D. Poisson. Recherches sur la probabilité des jugements. Paris. 1837.

[15] E. B. Wilson. Probable inference, the law of succession, and statistical inference. J. Amer. Statist. Assoc., Vol 27, pp. 209-212, 1927. 